\title{
¿YO TENÍA O YO TUVE? LA INFLUENCIA DEL ASPECTO LÉXICO-SEMÁNTICO EN LA ADQUISICIÓN DEL SISTEMA DE TIEMPO Y ASPECTO EN EL ESPAÑOL COMO LENGUA EXTRANJERA
}

\author{
Leyla Hasbún Hasbún
}

\begin{abstract}
RESUMEN
El presente estudio analiza la Hipótesis del Aspecto (Andersen 1986, 1991) la cual predice que en las etapas iniciales de la adquisición de una lengua, el uso de la morfología que marca el tiempo y el aspecto es fuertemente inflenciada por el aspecto léxico-semántico. Ochenta hablantes nativos del inglés que estudiaban español como lengua extranjera participaron en una prueba escrita donde los sujetos contaban un segmento de una película. El estudio examinó la distribución de la morfología verbal (perfectiva e imperfectiva) en las cuatro categorías léxicosemánticas y en cuatro niveles de competencia lingüística. Los resultados indican que hay una asociación muy fuerte entre la morfología perfectiva y los verbos de consecución y de cumplimiento y entre la morfología imperfectiva y los verbos estativos. Contrario a lo que dice la hipótesis, esta asociación se hizo más fuerte a medida que aumentaba el nivel de competencia.
\end{abstract}

\begin{abstract}
This study tests the Aspect Hypothesis (Andersen 1986, 1991) which predicts that in the beginning stages of acquisition, the use of tense and aspect morphology is influenced by lexical aspect. Eighty English-speaking learners of Spanish participated in a written film retell task. The study examined the distribution of verbal morphology (preterite and imperfect) across lexical aspectual class as a function of proficiency level. The results show that there is a significant association between perfective morphology and telic verbs and imperfective morphology and state verbs. Contrary to the predictions, this association becomes stronger with increased proficiency.
\end{abstract}

Uno de los rasgos más difíciles de aprender en el español como lengua extranjera es la distinción aspectual perfectivo/imperfectivo, que se manifiesta en el contraste entre el pretérito perfecto simple y el pretérito imperfecto. Este contraste ha sido objeto de numerosos estudios descriptivos y pedagógicos (Bello 1928; Bull 1960, 1965; Bolinger 1963; Ramsey 1967; Guitart 1978; Gili Gaya 1979; Fenwick 1980; Luján 1981; Sánchez 1981; Lunn 1985; Ozete 1988; Rojo 1988; De Mello 1989; Rodríguez Espiñeira 1990; Reyes 1990a, 1990b; 
Moure 1991). Sin embargo, existen muy pocos trabajos sobre la adquisición del tiempo y el aspecto en el español como segunda lengua (Chaston 1987), o como lengua extranjera (Andersen 1986, 1991; García y Putte 1988; Ramsay 1990; Hasbún 1995).

El objetivo principal de la presente investigación es examinar algunos factores que pueden determinar la adquisición del sistema de tiempo y aspecto del español (STAE). Probablemente, la mejor manera de alcanzar esta meta es observar el interlenguaje, es decir, el lenguaje que producen los aprendices de una segunda lengua mientras están en el proceso de adquisición. De esta forma, es posible generar el conocimiento que nos permita una mejor comprensión de la adquisición del lenguaje en general, y al mismo tiempo, la implementación de prácticas pedagógicas bien fundamentadas que hagan el proceso de enseñanza-aprendizaje más eficiente.

Antes de entrar a discutir las hipótesis planteadas en este trabajo, conviene aclarar el significado de los términos: "tiempo", "aspecto gramatical" y "aspecto léxico-semántico", principalmente para distinguir los dos últimos, ya que los conceptos de tiempo y aspecto gramatical son de todos conocidos y no presentan mayor confusión. Se entiende por "tiempo" del verbo la localización de un evento en el tiempo (Comrie 1985). Es decir, estamos hablando de las nociones de presente, pasado y futuro.

El aspecto gramatical se refiere a ciertas distinciones que son marcadas obligatoriamente en una lengua. Según Comrie (1976), se entienden por aspecto los diferentes modos de representar la constitución interna de un evento. En español, los aspectos gramaticales que mayor importancia tienen son el perfectivo y el imperfectivo. Prototípicamente, al utilizar las formas imperfectivas, "la atención del hablante se fija en el transcurso o continuidad de la acción, sin que le interesen el comienzo o el fin de la misma" (Gili Gaya 1961: 148). En contraste, al utilizar las formas perfectivas, "resalta la delimitación temporal" (Gili Gaya 1961: 149).

Por el contrario, el "aspecto léxico-semántico", llamado también Aktionsart, modo de la acción (Gili Gaya 1991) o aspecto situacional (Smith 1991), no es morfológico ni se puede manipular como el tiempo y el aspecto verbales. Este se desprende del contenido semántico inherente al verbo o a todo el predicado verbal. De acuerdo con Vendler (1967), el aspecto léxico-semántico establece cuatro categorías: verbos o predicados verbales estativos, de actividad, de consecución y de cumplimiento (la terminología en español es la empleada por Russinovich-Solé 1990). Los verbos estativos persisten a través del tiempo, por ejemplo, "vivir", "existir", "tener" y "saber". Los verbos de actividad tienen duración inherente pero no tienen un punto final en sí mismos, por ejemplo, "cantar”, "correr", "caminar" o "nadar". Los verbos de consecución, como los verbos de actividad, tienen duración inherente pero, además, tienen un final en sí mismos, por ejemplo, "pintar un cuadro", "caminar una milla", "escribir una carta" o "cantar una canción". Los verbos de cumplimiento ocurren en forma instantánea y representan un evento puntual o el principio o final de un evento o estado, por ejemplo, "reconocer", "encontrar", "llegar", "empezar" o "salir".

La convergencia del tiempo, el aspecto gramatical y el aspecto léxico-semántico se puede elucidar al analizar los siguientes ejemplos: 


\section{Cuadro 1}

Convergencia de los conceptos de tiempo, aspecto gramatical y aspecto léxico-semántico

\begin{tabular}{lllll}
\hline Ejemplo & Tiempo del Verbo & & Aspecto Gramatical & Aspecto Léxico-Semántico \\
\cline { 2 - 3 } salir del café & & & & cumplimiento \\
sale del café & presente & imperfectivo & cumplimiento \\
salió del café & pasado & perfectivo & cumplimiento \\
salía del café & pasado & imperfectivo & cumplimiento \\
\hline
\end{tabular}

Roger W. Andersen $(1986,1990,1991,1993)$ ha llevado a cabo una serie de investigaciones para analizar la adquisición del STAE por parte de hablantes nativos y no nativos. Andersen (1991) argumenta que sus datos tienden a corroborar los hallazgos de un número considerable de estudios sobre la adquisición de la lengua materna (Bronckart y Sinclair 1973; Antinucci y Miller 1976; Bloom, Lifter y Hafitz 1980), de una lengua extranjera, (Kumpf 1984; Economides 1985; Rothstein 1985; Nixon 1986), y de lenguas criollas (Bickerton 1975, 1981; Givón 1982), que indican que "la morfología verbal se desarrolla primero para expresar nociones aspectuales tales como 'completivo' o 'perfectivo', y sólo más tarde se utiliza para expresar el tiempo, presente o pasado" (Andersen 1986: 115). Mediante el uso de datos provenientes de un grupo de estadounidenses que aprendían español como segunda lengua en Puerto Rico así como datos recogidos por sus estudiantes, Andersen sentó las bases para un grupo de hipótesis relacionadas con la adquisición de un segundo idioma. Él propone lo siguiente:

\footnotetext{
Durante las primeras etapas de la adquisición de una lengua, la morfología del verbo es usada para codificar las distinciones léxico-semánticas inherentes en el verbo solamente, y no el tiempo o aspecto gramaticales (Andersen 1991: 307, traducción de la autora).
}

En otras palabras, lo que Andersen ha observado es que los aprendices principiantes muestran inicialmente una tendencia a asociar cierta morfología verbal con ciertos tipos de verbos, más específicamente, la morfología del pretérito con verbos de cumplimiento y de consecución, la morfología del imperfecto con verbos estativos y la morfología del progresivo con verbos de actividad. No es sino hasta más tarde en su desarrollo lingüístico que empiezan a utilizar la morfología del pretérito con los verbos de actividad y de estado y la morfología del imperfecto con los verbos de cumplimiento y consecución, como lo hacen los hablantes nativos.

En estudios posteriores Andersen (1993) trata de encontrar principios que puedan explicar el por qué de este comportamiento de los aprendices. Asevera que las asociaciones entre ciertas inflexiones y ciertas categorías léxico-semánticas son facilitadas por el lazo semántico que existe entre ambas. Por ejemplo, conceptualizamos la noción de pasado como algo que se refiere a un evento que ya ha terminado. Igual afirmación podemos hacer del aspecto perfectivo. Estos dos conceptos, tiempo pasado y aspecto perfectivo, se asocian fácilmente a frases verbales de consecución o cumplimiento las cuales, por su propia naturaleza, describen eventos que han terminado una vez 
que ocurren. No persisten en el tiempo como los estados o las actividades. Por lo tanto, en el proceso de adquisición de un complejo sistema de tiempo y aspecto verbales de una lengua, el uso de cierta morfología emerge primero en aquellos verbos que pertenecen a la categoría léxico-semántica donde es más fácil o más lógico percibir la conexión. Gradualmente, el uso de la morfología se extiende a otras categorías donde el enlace semántico entre la inflexión y la categoría léxico-semántica es más débil, y por consiguiente, es más difícil o menos lógico ver la conexión.

Otra posible explicación para las semejanzas en el comportamiento linguiístico de los aprendices de diferentes lenguas es que la morfología de la lengua meta tienda a distribuirse de manera similar a la morfología que utilizan los aprendices (Andersen 1992, 1993; Andersen y Shirai 1994). Si los hablantes nativos de una lengua en realidad también tienen un sesgo en la distribución de la morfología verbal en las cuatro categorías léxico-semánticas, esto explicaría en parte el por qué del comportamiento conservador de los aprendices. Por consiguiente, las dos explicaciones podrían respaldar los datos. Por un lado, ciertas asociaciones son adquiridas más temprano y más fácilmente ya que son congruentes. Por otro lado, la información linguiística o input que reciben los aprendices de parte de los hablantes nativos ratifica la misma distribución.

Con el fin de probar estas hipótesis, Bardovi-Harlig y Reynolds (1995) realizaron un estudio de 182 adultos que aprendían el inglés como segunda lengua en los Estados Unidos. Querían corroborar si el aspecto léxico-semántico determinaba la adquisición del tiempo pasado en el inglés. Encontraron que este juega un papel muy importante en el uso del pasado, pues los estudiantes mostraron un uso temprano y correcto del pasado con verbos de cumplimiento y de consecución, mientras el uso de la misma forma con verbos estativos y de actividad fue menos frecuente y mucho más tardío.

Bardovi-Harlig y Bergström (1996) estudiaron a un grupo de 20 adultos que aprendían el inglés como segunda lengua y 20 adultos que aprendían el francés como lengua extranjera. Encontraron influencia del aspecto léxico-semántico tanto en el uso del pasado simple en el inglés como en el del passé composé en el francés. En el primer caso, el pasado apareció primero en los verbos de cumplimiento y hasta después fue usado, primero, con los de consecución y, finalmente, con los de actividad. En cuanto al imperfecto del francés, los sujetos de la muestra lo usaron exclusivamente con los verbos estativos. Estos dos estudios apoyan las hipótesis de Andersen.

En cuanto a la adquisición del español, Ramsay (1990) examinó datos orales de 30 aprendices de español como lengua extranjera y concluyó que la adquisición del aspecto gramatical se desarrolla en cuatro etapas. En la primera, la mayoría de los verbos toman formas que divergen de aquellas usadas por los hablantes nativos (nótese que no se usa el adjetivo "incorrectas" ya que se considera que el aprendiz está pasando por una serie de etapas necesarias para la adquisición del sistema). En la segunda, un pequeño número de verbos, en su mayoría de cumplimiento, toma la morfología perfectiva. En la tercera, aparecen algunos verbos estativos con morfología imperfectiva. Al mismo tiempo, un mayor porcentaje de verbos de consecución y de cumplimiento recibe morfología perfectiva. En la cuarta, se lleva a cabo la adquisición de las formas perfectivas e imperfectivas. Estas etapas son consistentes con las hipótesis de Andersen (1986). Ramsay (1990: 173), sin embargo, opina que la frecuencia con que el aprendiz tiene contacto con las formas a través de la lectura o de lo que escucha (input frequency) y el uso de fórmulas o expresiones aprendidas de memoria, nos dan una explicación más plausible de este fenómeno que la propuesta por Andersen (1986). 
El presente estudio se propone contestar dos preguntas. La primera es: ¿cuál es la distribución del uso de la morfología correspondiente al presente, al pretérito imperfecto y al pretérito perfecto simple a través de las cuatro clases léxico-semánticas de verbos en las composiciones de un grupo de estudiantes que aprenden el español como lengua extranjera? La segunda pregunta es: si en realidad existe una asociación entre algunas clases léxico-semánticas y el uso de la morfología que marca tiempo y aspecto verbal, ¿se vuelve esta asociación más débil a medida que aumenta el nivel de competencia lingüística de los aprendices?

\section{Metodología}

Sujetos: En el estudio participaron 80 adultos hablantes nativos del inglés que aprendían español como lengua extranjera en una universidad. Se utilizaron cuatro grupos con 20 estudiantes cada uno, que representaban los cuatro primeros semestres del programa y que, para efectos de este estudio, fueron identificados como nivel 1 , nivel 2 , nivel 3 y nivel 4 , respectivamente.

Procedimiento: Se siguió el procedimiento utilizado por Bardovi-Harlig (1995) y Bardovi-Harlig y Bergström (1996). Los sujetos vieron un segmento de 8 minutos de la película del cine mudo titulada Modern Times. Después dé ver el segmento, se les pidió que contaran la historia por escrito y que dieran el mayor número de detalles posibles. Se les instó a adivinar cuando no estuvieran muy seguros acerca de la estructura o el léxico y se les aseguró que su desempeño en la tarea no influiría de manera alguna sobre su nota en el curso. El tiempo para realizar la prueba fue de 40 minutos. Para evitar que los estudiantes utilizaran el presente histórico, se les pidió que empezaran su relato con la frase "Había una vez. ..".

\section{Análisis}

La primera etapa del análisis realizado fue de tipo morfológico. Se leyeron las composiciones escritas por los aprendices y todos los predicados verbales fueron clasificados de acuerdo con la morfología que marca el tiempo y el aspecto gramatical. Esta primera clasificación fue de carácter descriptivo, por lo que no se juzgó si la morfología empleada era correcta o no de acuerdo con el contexto. Se incluyeron en el corpus todos los verbos que reflejaban conocimiento de las diferentes formas verbales, incluyendo regularizaciones tales como "venió" por "vino" o "dijó" por "dijo". No se prestó atención a la ortografía, incluyendo acentos ortográficos, siempre y cuando el error no interfiriera con el significado. Las formas verbales incomprensibles o aquellas que por su carácter podían ser solamente adivinadas o interpretadas por un profesor bilingüe fueron descartadas. Finalmente, como la concordancia es un problema adicional en la adquisición del español y las investigaciones indican que es adquirida independientemente del STAE (Andersen 1991: 309), se hizo caso omiso de estos errores.

La segunda etapa en el análisis consistió en asignarle una categoría léxico-semántica a cada predicado mediante el uso de una serie de pruebas diseñadas por Clements (1985) para el español. Por lo tanto, todos los predicados fueron clasificados como verbos estativos, de actividad, de consecución o de cumplimiento. 
Después de que todos los predicados fueron clasificados morfológica y semánticamente, se procedió a hacer un análisis de distribución, primero por sujeto y luego por grupo.

Finalmente, para determinar si realmente existía una asociación entre el aspecto léxico-semántico y la morfología que marca el tiempo y el aspecto gramatical, se realizó un análisis estadístico, utilizando la prueba Chi Cuadrada. Como esta prueba implica el uso de observaciones independientes, para este análisis se reorganizaron los datos y se reportó el uso más frecuente de cada uno de los individuos.

\section{Resultados y discusión}

El cuadro 2 presenta los resultados con relación a la morfología verbal usada por los estudiantes en los cuatro niveles definidos.El objetivo primordial es describir qué es lo que los aprendices realmente hacen en diferentes etapas de su adquisición, y no evaluar si lo que hacen es correcto o incorrecto.

Cuadro 2

Uso de las diferentes formas verbales según niveles: Porcentajes grupales

\begin{tabular}{lllll}
\hline Nivel & $\begin{array}{l}\text { Promedio de frases } \\
\text { usadas }\end{array}$ & $\begin{array}{l}\text { Porcentaje de formas } \\
\text { estándar }\end{array}$ & $\begin{array}{l}\text { Porcentaje de } \\
\text { gerundios e infinitivos }\end{array}$ & $\begin{array}{l}\text { Porcentaje de } \\
\text { formas no estándar }\end{array}$ \\
\hline 1 & $\begin{array}{l}\mathrm{X}=29.2 \\
\mathrm{~S}=9.9\end{array}$ & $3.9 \%$ & $14.3 \%$ \\
2 & $\begin{array}{l}\mathrm{X}=27.0 \\
\mathrm{~S}=11.3\end{array}$ & $80.9 \%$ & $1.5 \%$ & $17.6 \%$ \\
3 & $\mathrm{X}=34.3$ & $0.9 \%$ & $7.1 \%$ \\
$\mathrm{~S}=11.4$ & $92.0 \%$ & & $3.8 \%$ \\
\hline
\end{tabular}

Estos datos reflejan un perfil general del interlenguaje de los estudiantes con respecto al uso de la morfología verbal. La primera columna presenta el nivel (semestre) de los estudiantes. La segunda columna muestra la longitud promedio de las composiciones, medida en número de frases usadas $(\mathrm{X})$, y la desviación estándar correspondiente (S). El número de frases tiende a aumentar con el nivel de competencia al igual que la desviación estándar, como es de esperarse. La tercera columna nos da los porcentajes de formas verbales que forman parte de la lengua española que fueron utilizadas por los estudiantes de cada nivel. Como se puede ver, hay más de diez puntos de diferencia al comparar los porcentajes de los niveles 1 y 2 con los porcentajes de los estudiantes en los niveles 3 , un posible indicador de desarrollo lingüístico. Es importante destacar que a pesar de que los verbos empleados tienen una forma 
gramaticalmente correcta, no siempre fueron usados apropiadamente de acuerdo con el contexto. Esta importante diferencia y las implicaciones pedagógicas que tiene han sido discutidas en la literatura (Bardovi-Harlig y Bofman 1989; Bardovi-Harlig 1992).

Finalmente, las dos últimas columnas resumen los intentos infructuosos de utilizar la morfología del español. La cuarta columna muestra los porcentajes de verbos no conjugados usados en contextos donde la conjugación es necesaria. Nótese que los porcentajes son muy pequeños en todos los niveles y que decrecen a medida que aumenta el nivel de competencia lingüística. La última columna presenta los porcentajes de formas verbales que no son interpretables. Una vez más, los porcentajes son estadísticamente bajos y tienden a decrecer.

El cuadro 2 resume un patrón lógico: las narraciones escritas por los aprendices se tornan más largas y el número de verbos correspondientes a la lengua española se incrementa a medida que aumenta la competencia linguiística de los estudiantes. Asimismo, el número de verbos anómalos decrece a medida que se desarrolla el interlenguaje. La única excepción a este patrón parecen ser las narraciones escritas en el nivel 2. Estas son un tanto más cortas que las escritas en el nivel 1, y tienen un porcentaje un poco más alto de formas incomprensibles. Sin embargo, existen al menos tres factores que pueden explicar este comportamiento.

En primer lugar, es posible que el grupo en el nivel 1 fuera excepcionalmente talentoso mientras que los estudiantes en el nivel 2 no estuvieran muy motivados a aprender. Durante una serie de conversaciones informales que la investigadora tuvo con los instructores de ambos grupos, estos expresaron opiniones que tienden a confirmar esta apreciación. Si esto fuera cierto, tendríamos al menos una explicación parcial al fenómeno. En segundo lugar, de acuerdo con los programas, las formas del pretérito perfecto y del imperfecto se enseñan precisamente durante el segundo semestre. La difícil tarea de tratar de integrar la nueva morfología a su interlenguaje pudo haber sido un factor que desalentara a los estudiantes. Además, los intentos que hicieron para utilizar la morfología del pasado pudo haber propiciado el incremento en el número de las formas no interpretables. Finalmente, es muy posible que los aprendices que formaron parte de esta muestra representen no cuatro, sino únicamente dos etapas de desarrollo linguístico. En la primera etapa tendríamos a los estudiantes en los niveles 1 y 2. Los del nivel 2 no muestran ningún incremento en su desarrollo, ya que están aún procesando la nueva morfología. Esto implica un largo proceso donde los aprendices deben probar sus propias hipótesis acerca de la lengua. En la segunda etapa tendríamos a los estudiantes de los niveles 3 y 4, quienes han alcanzado un nivel considerable de precisión y están capacitados para escribir narrativas más extensas y sofisticadas.

El cuadro 3 presenta un resumen de la distribución del uso de la morfología verbal en relación con cada una de las cuatro categorías léxico-semánticas: verbos estativos, de actividad, de consecución y de cumplimiento. La distribución está hecha por nivel de competencia. Para simplificar nuestro análisis, este cuadro incluye solamente los datos referentes al uso del pretérito, del imperfecto y del presente, es decir, las tres formas usadas con mayor frecuencia por los aprendices y las que nos ocupan en esta investigación. Cada casilla presenta el número de verbos que fueron marcados con el pretérito, el imperfecto o el presente. Sin embargo, para dar mayor objetividad al análisis, los porcentajes que se reportan en paréntesis fueron computados con base en el número total de verbos producidos por los aprendices (que incluye algunos casos del pretérito pluscuamperfecto, formas progresivas, infinitivos, gerundios y participios) y no en el número de verbos que aparecen en la tabla; por lo tanto, no suman necesariamente $100 \%$. 


\section{Cuadro 3}

Distribución de la morfología correspondiente al pretérito, el imperfecto y el presente en cada una de las categorías léxico-semánticas según niveles de competencia

\begin{tabular}{|c|c|c|c|c|c|}
\hline Nivel/Forma & Estativos & Actividad & Consecución & Cumplimiento & Total \\
\hline \multicolumn{6}{|l|}{ Nivel 1} \\
\hline Pretérito & $10(6.2 \%)$ & $3(2.45 \%)$ & $8(7.7 \%)$ & $0(0.0 \%)$ & 21 \\
\hline Imperfecto & $1(0.6 \%)$ & $0(0.0 \%)$ & $0(0.0 \%)$ & $0(0.0 \%)$ & 1 \\
\hline Presente & $149(92.6 \%)$ & $105(84.7 \%)$ & $85(81.7 \%)$ & $96(87.3 \%)$ & 435 \\
\hline Total & & & & & 457 \\
\hline \multicolumn{6}{|l|}{ Nivel 2} \\
\hline Pretérito & $23(19.0 \%)$ & $25(29.4 \%)$ & $41(37.6 \%)$ & $66(51.2 \%)$ & 155 \\
\hline Imperfecto & $39(32.2 \%)$ & $5(5.9 \%)$ & $3(2.8 \%)$ & $3(2.3 \%)$ & 50 \\
\hline Presente & $52(43.0 \%)$ & $44(51.8 \%)$ & $59(54.1 \%)$ & $52(40.3 \%)$ & 207 \\
\hline Total & & & & & 412 \\
\hline \multicolumn{6}{|l|}{ Nivel 3} \\
\hline Pretérito & $19(16.8 \%)$ & $64(48.1 \%)$ & $110(85.9 \%)$ & $238(90.5 \%)$ & 431 \\
\hline Imperfecto & $79(69.9 \%)$ & $27(20.3 \%)$ & $2(1.6 \%)$ & $6(2.3 \%)$ & 114 \\
\hline Presente & $11(9.7 \%)$ & $6(4.5 \%)$ & $15(11.7 \%)$ & $13(4.9 \%)$ & 45 \\
\hline Total & & & & & 590 \\
\hline \multicolumn{6}{|l|}{ Nivel 4} \\
\hline Pretérito & $37(17.9 \%)$ & $64(40.2 \%)$ & $140(84.4 \%)$ & $304(89.4 \%)$ & 545 \\
\hline Imperfecto & $126(60.9 \%)$ & $61(38.4 \%)$ & $9(5.4 \%)$ & $12(3.5 \%)$ & 208 \\
\hline Presente & $26(12.5 \%)$ & $9(5.7 \%)$ & $4 \quad(2.4 \%)$ & $4(1.2 \%)$ & 43 \\
\hline Total & & & & & 796 \\
\hline
\end{tabular}

Al analizar el nivel 1, se observa que de los 457 verbos considerados en este nivel, 435 presentan morfología correspondiente al presente, más del $90 \%$ de los verbos estativos y el $80 \%$ de las otras clases. Esto se debe primordialmente a que los alumnos no habían estudiado aún las formas pasadas cuando se recogió la muestra. No obstante, los aprendices emplearon otras 22 formas con un grupo limitado de verbos. De ellas, 21 fueron formas pretéritas de cuatro verbos de uso frecuente, a saber, "ser" y "tener" que se clasifican como estativos, "hablar" que se clasifica como de actividad, e "ir a" que se clasifica como de consecución. Los verbos "ser" e "ir" no son solamente comunes sino que también tienen forma pretérita idéntica, lo que pudo haber contribuido a su memorización. En cuanto a la única forma del imperfecto, ocurrió con el verbo estativo "ser". Contrario a las predicciones de Andersen (1986), en este primer nivel, los verbos de consecución y de cumplimiento no fueron los primeros en recibir morfología per- 
fectiva. Sin embargo, las formas del pretérito y del imperfecto que se encuentran en la muestra más bien parecen ser fórmulas memorizadas producidas por únicamente 6 de los 20 estudiantes, y no evidencia de la adquisición del STAE.

La diferencia más significativa entre los aprendices del primer y el segundo nivel parece ser la aparición del uso del pretérito perfecto y del imperfecto. En este nivel, de los 412 verbos analizados, 205 (49\%) recibieron morfología correspondiente al pasado (pretérito perfecto o imperfecto). Pareciera también que emerge un patrón de uso donde la mayoría de los verbos de cumplimiento reciben morfología perfectiva mientras que los estativos reciben imperfectiva, aunque en grado menor.

La distribución que presentan los estudiantes del nivel 3 es muy diferente. En este momento, de los 590 verbos empleados, 545 (92\%) reciben morfología del pretérito (perfecto o imperfecto). El patrón de distribución de morfología en las diferentes categorías léxico-semánticas que se observó en el nivel 2 parece reforzarse. El uso de la morfología perfectiva se extiende de los verbos de cumplimiento a los de consecución. Además, el uso de la morfología imperfectiva con los verbos estativos comienza a fortalecerse con los verbos de actividad.

De un total de 796 verbos analizados, los aprendices del nivel 4 usaron morfología del pretérito (perfecto o imperfecto) con 753 (95\%) de ellos. El porcentaje de uso de la morfología perfectiva con los verbos de cumplimiento y de consecución es similar al que se da en el nivel 3 . De hecho, pareciera que cuando los aprendices llegan al tercer nivel, ya han adquirido estas asociaciones, ya que la morfología perfectiva es usada en forma consistente tanto con los verbos de cumplimiento como con los de consecución. En cuanto a la morfología imperfectiva, los estudiantes del cuarto nivel muestran un uso un tanto menor con los verbos estativos pero algo más alto con las actividades, los de consecución y los de cumplimiento, lo que indica madurez lingüística.

Con el fin de determinar si las asociaciones observadas en el análisis anterior eran estadísticamente significativas, se llevó a cabo otro análisis por medio de la Chi cuadrada. En el nivel 1 , la $\mathrm{x}^{2}$ resultó significativa para las cuatro categorías léxico-semánticas ya que los estudiantes usan la morfología del presente casi exclusivamente. Para este grupo no hay una verdadera selección por lo que los resultados no son reveladores.

En el nivel 2, la $x^{2}$ no fue significativa para los estados y las actividades ya que no hay preferencia clara por ningún tipo de morfología. Para los verbos de consecución y de cumplimiento, sin embargo, la prueba arroja resultados significativos aunque el índice de asociación no es muy alto. Lo que la $x^{2}$ sugiere es el hecho de que los aprendices no usan la morfología imperfectiva con los verbos de consecución y de cumplimiento y que su selección de morfología está distribuida en forma equitativa entre las formas del presente y del pretérito perfecto simple.

En el nivel 3, la $x^{2}$ es significativa para las cuatro categorías léxico-semánticas. En cuanto a los verbos estativos, hay una asociación fuerte con las formas imperfectivas. Para las actividades, el índice de asociación es más débil. Lo que sugiere la prueba es el hecho de que no hay uso del presente pero sí un poco del imperfecto. En contraste, la asociación entre la morfología perfectiva y los verbos de consecución y de cumplimiento es muy fuerte.

En el nivel 4, la $x^{2}$ es significativa en todas las categorías léxico-semánticas. En los verbos estativos, hay una asociación fuerte con la morfología imperfectiva. En los verbos de actividad, la asociación es con las dos formas pretéritas (perfecto e imperfecto). En cuanto a los verbos de consecución y de cumplimiento, la asociación con las formas perfectivas es muy fuerte.

El siguiente cuadro resume los resultados de la prueba $\mathrm{x}^{2}$. 


\begin{abstract}
Cuadro 4
Asociación entre la preferencia de uso de la morfología del presente, el pretérito perfecto y el imperfecto y la categoría léxico-semántica según la $x^{2}$
\end{abstract}

\begin{tabular}{lllll}
\hline Nivel & Estativos & Actividad & Consecución & Cumplimiento \\
\hline Nivel 1 & Presente** & Presente** & Presente** & Presente** \\
Nivel 2 & Pres/Perf/Imp & Presente/Perf & Presente/Perf* & Presente/Perf* \\
Nivel 3 & Imperfecto* & Perfecto* & Perfecto** & Perfecto** \\
Nivel 4 & Imperfecto* & Perfecto* & Perfecto** & Perfecto** \\
\hline
\end{tabular}

*índice de asociación entre .50 y .74 (asociación significativa)

**índice de asociación entre .75 y superior (asociación muy fuerte)

Las preferencias representadas en el cuadro 4 parecen indicar que los aprendices del presente estudio han pasado por una serie de etapas en su adquisición del STAE, las cuales parecen estar influenciadas por la categoría léxico-semántica del verbo. En el nivel 1, los estudiantes usan el presente con todas las categorías. Esto no constituye una preferencia ya que ellos no han adquirido ninguna otra forma. En el nivel 2, se empieza a usar la morfología perfectiva, en especial con los verbos de consecución y de cumplimiento. En el nivel 3, el uso del pretérito con los verbos de consecución y de cumplimiento se fortalece, a la vez que emerge el uso de la morfología imperfectiva con los verbos estativos. En el nivel 4, las asociaciones hechas por los aprendices en el nivel 3 se intensifican. Con la excepción del nivel 1, estas etapas corresponden a las propuestas por Andersen (1991).

\title{
4. Conclusiones
}

El anterior análisis demuestra que la distribución del uso de la morfología verbal en las cuatro categorías léxico-semánticas tiene un claro sesgo. Es decir, aunque en el español se puedan emplear las formas del pretérito imperfecto y del pretérito perfecto simple con cualquier tipo de verbo, sea este un verbo estativo, de actividad, de consecución o de cumplimiento, los estudiantes tienden a asociar la morfología del pretérito perfecto simple con verbos de complimiento y de consecución y la morfología del pretérito imperfecto con verbos estativos. Un fenómeno similar ha sido observado en estudios sobre la adquisición de diversas lenguas (Andersen 1986 y 1991, Ramsay 1990, Hasbún 1995 para el español, Bardovi-Harlig 1992 y Bardovi-Harlig y Reynolds 1995 para el inglés, Bardovi-Harlig y Bergström 1996 y Bergström 1995 para el francés).

Todos estos estudios corroboran al menos la interpretación que hace Robison (1990) de las hipótesis de Andersen (1986) cuando afirma que "la morfología verbal se correlaciona con el aspecto léxico al menos durante una etapa en el desarrollo del interlenguaje (Robison 1990: 330, traducción de la autora). Sin embargo, no queda tan claro si los resultados de la presente investigación apoyan completamente la hipótesis original del aspecto de Andersen (1991). El presente estudio demostró que estadísticamente existe un sesgo. Sin embargo, el 
por qué esto ocurre no es tan evidente ya que la experiencia nos indica que desde las primeras etapas, los aprendices adultos que reciben educación formal parecen usar la morfología verbal para expresar diferencias de tiempo. En el aula de clase, los estudiantes son entrenados específicamente para hacerlo. Más tarde, sus sistemas maduran y los aprendices empiezan a manipular el aspecto gramatical y a desarrollar su potencial expresivo.

El análisis de la distribución de la morfología verbal en el habla y la escritura de los hablantes nativos es necesaria ya que podría contribuir a una mejor comprensión de hasta qué punto la información lingüística recibida es responsable de la distribución hallada en los aprendices. Una vez establecido el comportamiento de los hablantes nativos, se debe proceder a la elaboración de pautas pedagógicas que permitan al docente acelerar y facilitar la adquisición del STAE.

\section{Bibliografía}

Andersen, R. W. 1986. "El desarrollo de la morfología verbal en el español como segundo idioma". En: J. M. Meisel (ed.), 115-138.

1990. "Models, processes, principles and strategies: Second language acquisition inside and outside the classroom." En: B. VanPatten \& J. F. Lee (eds.), 45-68.

1991. "Developmental sequences: The emergence of aspect marking in second language acquisition." En: C. A. Ferguson \& T. Huebner (eds.), 305-24.

1993. "Four operating principles and input distribution as explanations for underdeveloped and mature morphological systems." En: K. Hyltenstam \& A. Vigorb (eds.), 309-39.

Andersen, R. W. y Shirai, Y. 1994. "Discourse motivations for some cognitive acquisition principles." SSLA 16, 133-56.

Antinucci, F. y Miller, R. 1976. "How children talk about what happened." Journal of Child Language 3, 169-89.

Bardovi-Harlig, K. 1992. "The relationship of form and meaning: A cross-sectional study of tense and aspect in the interlanguage of learners of English as a second language." Applied Psycholinguistics 13, 253-78.

1995. "A narrative perspective on the development of tense and aspect." Studies in Second Language Acquisition 17, 263-91.

Bardovi-Harlig, K. y Bofman, T. 1989. "Attainment of syntactic and morphological accuracy by advanced language learners." Studies in Second Language Acquisition 11, 17-34.

Bardovi-Harlig, K. y Reynolds, D.W. 1995. "The role of lexical aspect in the acquisition of tense and aspect." TESOL Quarterly 29, 107-31. 
Bardovi-Harlig, K. y Bergström, A. 1996. "Acquisition of tense and aspect in second language and foreign language learning: Learner narratives in ESL and FFL." The Canadian Modern Language Review 52, 308-29.

Bello, A. 1928. Gramática de la lengua castellana destinada al uso de los americanos: Edición crítica de Ramón Trujillo. Santa Cruz de Tenerife: Litografía A. Romero.

Bergström, A. 1995. The expression of past temporal reference by English-speaking learners of French. Disertación doctoral no publicada, The Pennsylvania State University

Bickerton, D. 1975. Dynamics of a Creole System. Cambridge: Cambridge University Press. 1981. Roots of Language. Ann Arbor: Karoma Publishers.

Bloom, L., Lifter, K. y J. Hafitz. 1980. "Semantics of verbs and the development of verb inflection in child language." Language 56, 386-412.

Bolinger, D. 1963. Reference and inference: Inceptiveness in the Spanish preterite. Hispania $46,128-35$.

Bronckart, J. P. y H. Sinclair. 1973. “Time, tense and aspect.” Cognition 2, 107-30.

Bull, W. 1960. Time, tense and the verb. Berkeley: University of California Press.

1965. Spanish for teachers. New York: The Ronald Press Co.

Chaston, J. M. 1987. Aspect choice in preterit and imperfect usage in the speech of MexicanAmerican college students in Texas: A sociolinguistic approach. Disertación doctoral no publicada, The University of Texas at Austin.

Clements, J. A. 1985. Verbal classification and verb class change in Spanish. Disertación doctoral no publicada, University of Washington.

Comrie, B. 1976. Aspect. New York: Cambridge University Press.

1985. Tense. New York: Cambridge University Press.

De Mello, G. 1989. "Some observations on Spanish verbal aspect.” Hispanic Linguistics 3, 123-9.

Eckman F.R., L. H. Bell, \& D. Nelson (eds.). 1984. Universals of second language acquisition. Rowley, Massachusetts: Newbury House.

Economides, P. J. 1985. The expression of tense and aspect in the English interlanguage of a Vietnamese child. Tesis de maestría no publicada, UCLA. 
Fenwick, S. 1980. Verbal aspect: Its form and function in contemporary Spanish. Disertación doctoral no publicada, The University of Iowa.

Ferguson, C. A. \& T. Huebner (eds.). 1991. Second language acquisition and linguistic theories. Amsterdam: John Benjamins.

García, E. y Putte, F. C. M. 1988. "The value of contrast: Contrasting the value of strategies." IRAL 26, 263-81.

Gili Gaya, S. 1961. Curso superior de sintaxis española. Barcelona: Vox.

1979. Estudios del lenguaje infantil. Barcelona: Bibliograph, S. A.

Givón, T. 1982. "Tense-aspect-modality: The creole prototype and beyond." En: P. Hopper (ed.), 115-63.

Guitart, J. M. 1978. "Aspects of Spanish aspect: A new look at the preterit/imperfect distinction.” En: M. Suñer (ed.), 132-68.

Hasbún, L. 1995. The role of lexical aspect in the acquisition of the tense/aspect system in L2 Spanish. Disertación doctoral no publicada, Indiana University.

Hopper, P. (ed.) 1982. Tense-Aspect: Between Semantics and Pragmatics. Amsterdam: John Benjamins.

Hyltenstam, K. \& A. Vigorb (eds.). 1993. Progression and regression in language. Cambridge: Cambridge University Press.

Kumpf, L. 1984. "Temporal systems and universality in interlanguage: A case study." En: F. R. Eckman, L. H. Bell, \& D. Nelson (eds.), 132-43.

Luján, M. 1981. "The Spanish copulas as aspectual indicators.” Lingua 54, 165-210.

Lunn, P. 1985. "The aspectual lens." Hispanic Linguistics 2, 49-61.

Meisel, J. M. (ed.). 1986. Adquisición de lenguaje. Frankfurt: Vervuert.

Moure, T. 1991. "El contenido aspectual telicidad en las cláusulas biactanciales del español". Verba, 18, 353-74.

Nixon, N. 1986. Tense/aspect in the English interlanguage of a native Mandarin speaker. Tesis de maestría no publicada, UCLA.

Ozete, O. 1988. "Focusing on the preterite and imperfect." Hispania 71, 687-91. 
Ramsay, V. 1990. Developmental stages in the acquisition of the perfective and the imperfective aspects by classroom L2 learners of Spanish. Disertación doctoral no publicada, University of Oregon.

Ramsey, M. L. 1967. A textbook of modern Spanish. New York: Holt, Rinehart and Winston.

Reyes, G. 1990a. "Tiempo, modo, aspecto e intertextualidad." Revista Española de Lingüística $20,17-53$.

1990b. "Valores estilísticos del imperfecto." Revista de Filología Española 70, 45-70.

Robison, E. 1990. "The primacy of aspect: Aspectual marking in English interlanguage." Studies in Second Language Acquisition 12, 315-30.

Rodríguez Espiñeira, M.J. 1990. "Clases de Aktionsart y predicaciones habituales en español." Verba 17, 171-210.

Rojo, G. 1988. "Temporalidad y aspecto en el verbo español." Lingüística Española Actual 10, 195-216.

Rothstein, G. 1985. The expression of temporality in the English interlanguage of a native Hebrew speaker. Tesis de maestría no publicada, UCLA.

Russinovich Solé, Y. 1990. "Valores aspectuales en el español." Hispanic Linguistics 4, 57-86.

Sánchez, V. 1981. "Un problema de semántica: neutralización presente/pasado en castellano". Studii si Cercetari Lingvistice 32, 613-20.

Smith, C. 1991. The parameter of aspect. Boston: Kluwer Academic Publishers.

Suñer, M. (ed.). 1978. Contemporary studies in Romance linguistics. Washington D.C.: Georgetown University Press.

VanPatten, B. \& J. F. Lee (eds.). 1990. Second language acquisition/Foreign language learning. Clevedon: Multilingual Matters.

Vendler, Z. 1967. "Verbs and time." En: Z. Vendler (ed.), 97-121.

Vendler, Z. (ed.). 1967. Linguistics and philosophy. New York: Cornell University Press. 